\title{
The Stellar Space Distributions of dA and gK Stars
}

\author{
D. N. Dawanas \& B. Hidayat \\ Department of Astronomy and Bosscha Observatory, Institute of \\ Technology, Bandung, Indonesia
}

\begin{abstract}
We present a case study on the stellar space distribution of stars with MK spectral types B8 to A2 (dA) and gG8 to gK2 (gK) in a sky region of 25 square degrees, centred at the galactic coordinate $l=4^{\circ}$ and $b=+12^{\circ}$. Two hundred and forty-eight (248) stars with MK type of B8-A2 and gG8-gK2 were identified in this field. The space density distributions of these stars were obtained by employing the Matrix and Malmquist methods. The relative density of B8-A2 stars tends to be similar to that of gG8-gK2 stars, which implies that the two groups of stars occupy the same space volume in the galaxy. The comparison with those of Field-3 $\left(l=0.8^{\circ}, b=-10^{\circ}\right)$ show that the space density distribution of the two groups of stars above and below the galactic plane is not the same. Above the galactic plane, the space density of the $\mathrm{gK}$ stars is higher than those of dA stars, whereas below the galactic plane the density of dA stars is higher than for the gK stars. For both groups, the maximum space density occurs at around $30-40 \mathrm{pc}$ from the galactic plane.
\end{abstract}

\section{Introduction}

As part of our continuing interest in the structure, kinematics and evolution of our galaxy, we have underaken studies of specially chosen galactic regions. One of these is the meridional section in the southern sky. The study of the space distribution of the B8-A2 (dA) and gG8-gK2 (gK) stars is of particular interest. In the direction of the galactic centre and anti-centre, McCuskey (1965; 1970) has found that the B8-A0 main sequence stars and G8-K3 giant stars are concentrated around the sun in the galactic plane and decrease in number away from it. In a preliminary study of the distributions of $\mathrm{dA}$ and $\mathrm{gK}$ stars in the direction of the galactic centre, Hidayat (1970) indicated that the $\mathrm{dA}$ and $\mathrm{gK}$ stars occupy the same volume and have similar relative distributions. As the ages of $\mathrm{dA}$ and $\mathrm{gK}$ stars are around $1-5 \times 10^{6} \mathrm{yr}$, and they are concentrated in the same region, they are thought to represent the fossil of a spiral arm. Therefore, studying the space distribution of $\mathrm{dA}$ and $\mathrm{gK}$ stars can be expected to give information about the fossil spiral arm of the galaxy.

In the present study we determine the stellar space density of the two groups of stars in a direction near the galactic centre, at $l=4^{\circ}$ and $b=+12^{\circ}$. This region is known as the Groningen Variable Star Field-2 (Field-2). The results of this study, when combined with earlier results for the same galactic longitudes 
should provide information on the cross sectional distribution of stars in the plane perpendicular to the galactic plane in the galactic-centre direction.

\section{Observational Data}

The spectral and direct stellar images of the field were obtained with the 28 inch Schmidt telescope at the Bosscha Observatory. The spectra of the stars were recorded on Kodak IIa-O plates with exposure times of 4, 20 and $60 \mathrm{~min}$. To classify the spectra of the stars we used the criteria given by Nassau \& Seyfert (1946). From this classification we obtained a total of $138 \mathrm{dA}$ and $110 \mathrm{gK}$ stars. The limiting magnitude of this survey was approximately $V=12.10 \mathrm{mag}$. The $V$ and $B$ magnitudes of the Johnson and Morgan (1953) system were obtained from three Kodak 103a-D plates taken through a Schott GG 11 filter and three Kodak 103a-O plates taken through a Schott GG 13 filter. All stars for which spectra have been classified were measured on each plate. The probable errors of these measurements are 0.09 and $0.10 \mathrm{mag}$ for $V$ and $B-V$ respectively.

Inspection of the deep red and blue Bosscha Schmidt photometric plates did not show any appreciable patchiness in the surface distribution of interstellar material. Therefore we assume that the variations of interstellar absorption are the same throughout the area. The evaluation of the interstellar reddening has been made from photoelectric data given by Wehinger \& Hidayat (1973).

\section{Space Density Analysis}

To analyze the stellar space density we used the Matrix method described by Dolan (1974). For comparison we employed the Malmquist numerical method.

A summary of the star counts for each $1.0 \mathrm{mag}$ interval in the surveyed area is given in Table I. The absolute magnitude used in this study is the average absolute magnitude for each group and the absolute magnitudes for each subclass are taken from Schmidt-Kaler (1982). From this averaging we have $M_{V}=\mathbf{0 . 7 4}$ for $\mathrm{dA}$ stars and $M_{V}=0.68$ for $\mathrm{gK}$ stars.

The resultant space densities, corrected for interstellar absorption from both methods are given in Table II. In general, both methods produce similar results. We average values of these two results as shown in column 6 for dA stars and in column 7 for $\mathrm{gK}$ stars. From this averaging we found that the space densities of $\mathrm{dA}$ stars tend to be similar to those of $\mathrm{gK}$ stars, which implies that the two groups of stars occupy the same space volume in the galaxy.

In order to exhibit the density variation with respect to the distance from the galactic plane, the $r$-values in column 1 of Table II was transformed into $z$-values, where $z$ is the distance from the galactic plane, and the results are shown in column 8 of the same table.

\section{Discussion}

Other regions of high stellar concentrations have been detected by several authors, for example by McCuskey (1956) in LF's regions, Upgren (1963) in the North Galactic Pole region, Drilling (1968) in 15 LF regions and Stegman \& 


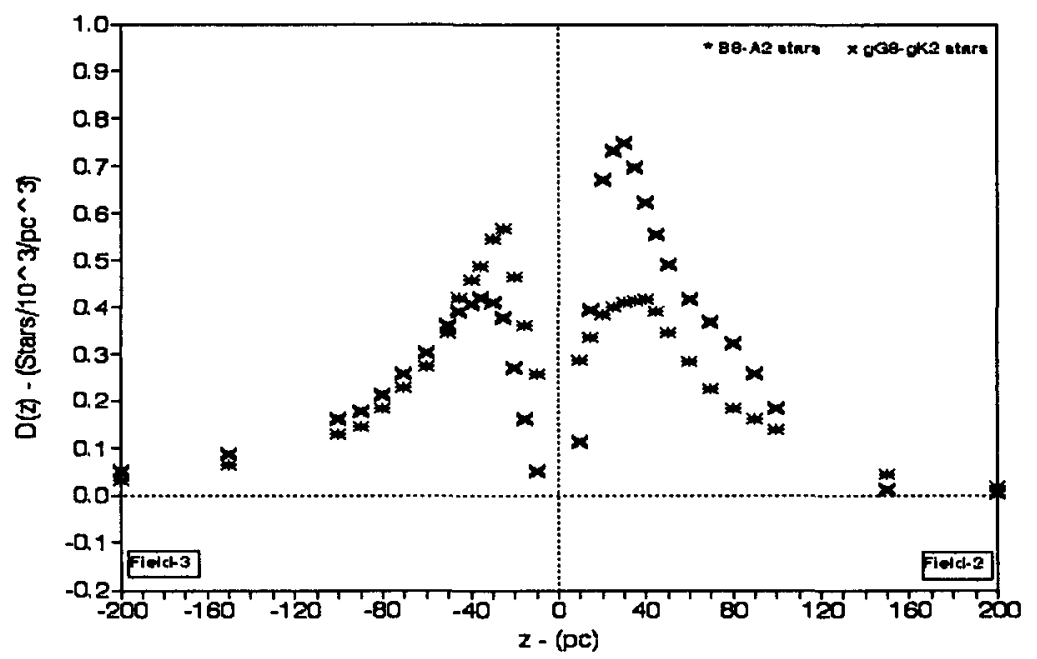

Figure 1. Space density distribution of $\mathrm{dA}$ and $\mathrm{gK}$ stars in the direction of Field-2 and Field-3.

Fitzgerald (1973) in the Vela region. All of these studies showed that the concentration of $\mathrm{dA}$ and gK stars are located between 500 to $800 \mathrm{pc}$, while the result of our study in Field-2 shows that the high stellar concentrations are found between 150 to $250 \mathrm{pc}$. Comparisons of the results obtained by the mentioned authors with that of Field-2, shows that the density of the dA and gK stars is higher in the direction of the galactic centre.

It is interesting to compare the present result with the result obtained for the Field-3 region with $l=0.8^{\circ}$ and $b=-10^{\circ}$ (Hidayat \& Radiman 1973). Fig. 1 shows the comparison. From this figure we can see that the relative space density distribution of $\mathrm{dA}$ and $\mathrm{gK}$ stars above and below the galactic plane is not symmetric. Above the galactic plane, the space density distribution of $\mathrm{gK}$ stars is higher than that of $\mathrm{dA}$ stars, whereas below the galactic plane the $\mathrm{dA}$ star is higher than $\mathrm{gK}$ stars. The maximum space density distribution for both groups above and below the galactic plane is almost the same, at around 30 pc from the galactic plane. These results differ from those of McCuskey (1965; 1970). According to McCuskey, both groups of stars are concentrated around the sun in the galactic plane. It would be instructive to compare the space density distribution in other directions in order to ascertain the differences and in particular to know the position of the sun with respect to the galactic plane (see Stobie \& Ishida 1987; Creze 1993). The result described above can be accounted for, if the distribution of stars around the sun is not uniform. This will be part of our future study. 
Table I : Observed distribution of $\mathrm{dA}$ and $\mathrm{gK}$ stars

\begin{tabular}{|r|r|r|}
\hline $\mathbf{V}$ & $\mathbf{d A}$ & \multicolumn{1}{|c|}{$\mathbf{g K}$} \\
\hline 5.5 & 1 & 1 \\
6.5 & 2 & 5 \\
7.5 & 8 & 11 \\
8.5 & 16 & 22 \\
9.5 & 32 & 51 \\
10.5 & 55 & 18 \\
11.5 & 24 & 3 \\
\hline
\end{tabular}

Table II: Space density variations of $\mathrm{dA}$ and $\mathrm{gK}$ stars as a function of distance from the sun $D(r)$ (in stars per $10^{3} \mathrm{pc}^{3}$ ) and as a function of distance from the galactic plane $D(z)$ (in stars per $10^{3} \mathrm{pc}^{3}$ ) calculated from Matrix and Malmquist methods.

\begin{tabular}{|c|c|c|c|c|c|c|c|c|c|}
\hline \multirow{3}{*}{$\begin{array}{c}\mathbf{r} \\
\text { (pe) }\end{array}$} & \multicolumn{6}{|c|}{$D(r)$ or $\mathbf{D}(z)$} & \multirow{2}{*}{\multicolumn{2}{|c|}{$\begin{array}{c}\text { Averages of } \\
D(r) \\
\text { or } \mathbf{D}(z)\end{array}$}} & \multirow{3}{*}{$\begin{array}{c}\mathbf{z} \\
(\mathrm{pc})\end{array}$} \\
\hline & \multicolumn{3}{|c|}{$\overline{B 8}-A 2(d A)$} & \multicolumn{3}{|c|}{ gG8 - gK2 (gK) } & & & \\
\hline & Matrix & Method & $\begin{array}{l}\text { Malmquist } \\
\text { Method }\end{array}$ & Matrix & Method & $\begin{array}{c}\text { Malmquist } \\
\text { Method }\end{array}$ & $\mathbf{d A}$ & gK & \\
\hline 50 & 0.3932 & \pm 0.4763 & 0.1862 & 0.1206 & \pm 0.4490 & 0.1465 & 0.2897 & 0.1335 & 10.4 \\
\hline 100 & 0.3498 & \pm 0.5137 & 0.4335 & 0.5180 & \pm 0.5938 & 0.9156 & 0.3916 & 0.7168 & 20.8 \\
\hline 150 & 0.3717 & \pm 0.2708 & 0.4500 & 0.7252 & \pm 0.3474 & 0.7813 & 0.4109 & 0.7533 & 31.2 \\
\hline 200 & 0.4327 & \pm 0.1835 & 0.4005 & 0.5612 & \pm 0.2070 & 0.6359 & 0.4166 & 0.5985 & 41.6 \\
\hline 250 & 0.3205 & \pm 0.1081 & 0.3363 & 0.4097 & \pm 0.1264 & 0.5178 & 0.3284 & 0.4638 & 52.0 \\
\hline 300 & 0.2745 & \pm 0.0865 & 0.2652 & 0.3970 & \pm 0.0977 & 0.4045 & 0.2698 & 0.4008 & 62.4 \\
\hline 350 & 0.2229 & $\pm \quad 0.0604$ & 0.1940 & 0.3900 & \pm 0.0711 & 0.3135 & 0.2085 & 0.3518 & 72.8 \\
\hline 400 & 0.1824 & \pm 0.0406 & 0.1648 & 0.3746 & \pm 0.0464 & 0.2401 & 0.1736 & 0.3073 & 83.2 \\
\hline 450 & 0.1649 & \pm 0.0340 & 0.1365 & 0.2918 & \pm 0.0374 & 0.1666 & 0.1507 & 0.2292 & 93.6 \\
\hline 500 & 0.1474 & \pm 0.0274 & 0.1082 & 0.2089 & $\pm \quad 0.0283$ & 0.0932 & 0.1278 & 0.1511 & 104.0 \\
\hline 550 & 0.1299 & \pm 0.0209 & 0.0813 & 0.1261 & \pm 0.0193 & 0.0743 & 0.1056 & 0.1002 & 114.4 \\
\hline 600 & 0.1124 & \pm 0.0143 & 0.0708 & 0.0232 & \pm 0.0082 & 0.0596 & 0.0916 & 0.0414 & 124.7 \\
\hline 650 & 0.0964 & \pm 0.0127 & 0.0604 & 0.0198 & \pm 0.0072 & 0.0448 & 0.0784 & 0.0323 & 135.1 \\
\hline 700 & 0.0803 & \pm 0.0108 & 0.0499 & 0.0164 & \pm 0.0061 & 0.0300 & 0.0651 & 0.0232 & 145.5 \\
\hline 750 & 0.0641 & \pm 0.0090 & 0.0395 & 0.0130 & $\pm \quad 0.0050$ & 0.0153 & 0.0518 & 0.0142 & 155.9 \\
\hline 800 & 0.0480 & \pm 0.0071 & 0.0221 & 0.0096 & \pm 0.0040 & 0.0073 & 0.0351 & 0.0085 & 166.3 \\
\hline 850 & 0.0319 & \pm 0.0053 & 0.0200 & 0.0062 & \pm 0.0029 & 0.0064 & 0.0260 & 0.0063 & 176.7 \\
\hline 900 & 0.0157 & \pm 0.0034 & 0.0180 & 0.0028 & \pm 0.0018 & 0.0055 & 0.0169 & 0.0042 & 187.1 \\
\hline 950 & & & 0.0159 & & & 0.0046 & 0.0159 & 0.0046 & 197.5 \\
\hline 1000 & & & 0.0138 & & & 0.0037 & 0.0138 & 0.0037 & 207.9 \\
\hline 1250 & & & 0.0035 & & & 0.0003 & 0.0035 & 0.0003 & 259.9 \\
\hline 1500 & & & 0.0015 & & & 0.0002 & 0.0015 & 0.0002 & 311.9 \\
\hline 1750 & & & 0.0006 & & & 0.0000 & 0.0006 & 0.0000 & 363.8 \\
\hline 2000 & & & 0.0001 & & & 0.0000 & 0.0001 & 0.0000 & 415.8 \\
\hline
\end{tabular}




\section{Acknowledgements}

We acknowledge with many thanks the support from the Warner and Swasey Observatory and from the Leids-Kerkhoven-Bosscha Foundation for plates used in the present study.

\section{References}

Creze M., 1993, in Workshop on Data Process for Galactic Structure, eds. A. G. D. Philip, B. Hauck \& A. R. Upgren, Davis Press, New York, p.109

Dolan J. F., 1974, A\&A, 35, 105

Drilling J. S., 1968, AJ, 73, 590

Hidayat B., 1970, Publ. Bosscha Obs. no. 4

Hidayat B. \& Radiman I., 1973, Con. Bosscha Obs. no. 46

Johnson H. L. \& Morgan W. W., 1953, ApJ, 117, 303

McCuskey S. W., 1956, ApJ, 123, 458

McCuskey S. W., 1965, in Galactic Structure, eds. A. Blaauw \& M. Schmidt, Univ, of Chicago Press, p. 1

McCuskey S. W., 1970, IAU Symp. No.38, eds. W. Becker \& G. Contopoulos, Reidel Publishing Co., p. 189

Nassau J. J. \& Seyfert C. K., 1946, ApJ, 103, 117

Schmidt-Kaler T. H., 1982, in Lanndolt Bronstein Catalogue, Vol. VI/2b, SpringerVerlag, Berlin

Stegman J. E. \& FitzGerald P. M., 1973, JRAS, Canada, 66, 303

Stobie R. S. \& Ishida K., 1987, AJ, 93, 624

Upgren A., 1963, AJ, 68, 475

Wehinger P. A. \& Hidayat B., 1973, AJ, 78, 401.

Note: While preparing this paper we noted that the Palomar-Groningen Feild 3 has been studied by $\mathrm{Y}$. K. Ng et al. (preprint, Leiden Observatory) but with a quite different approach. 